\title{
QUEEN'S
UNIVERSITY
BELFAST
}

\section{Heating a torus with number theory}

Pausinger, F., \& Steinerberger, S. (2019). Heating a torus with number theory. Mathematics Magazine, 92(2), 99-106. https://doi.org/10.1080/0025570X.2018.1546507

\section{Published in:}

Mathematics Magazine

\section{Document Version:}

Peer reviewed version

\section{Queen's University Belfast - Research Portal:}

Link to publication record in Queen's University Belfast Research Portal

\section{Publisher rights}

(c) 2018 Mathematical Association of America. This work is made available online in accordance with the publisher's policies. Please refer to any applicable terms of use of the publisher.

\section{General rights}

Copyright for the publications made accessible via the Queen's University Belfast Research Portal is retained by the author(s) and / or other copyright owners and it is a condition of accessing these publications that users recognise and abide by the legal requirements associated with these rights.

Take down policy

The Research Portal is Queen's institutional repository that provides access to Queen's research output. Every effort has been made to ensure that content in the Research Portal does not infringe any person's rights, or applicable UK laws. If you discover content in the Research Portal that you believe breaches copyright or violates any law, please contact openaccess@qub.ac.uk. 


\title{
HEATING A TORUS WITH NUMBER THEORY
}

\author{
FLORIAN PAUSINGER \& STEFAN STEINERBERGER
}

Suppose you live on a torus and want to heat it as efficiently as possible with a finite number of identical heat sources. What configuration is optimal and how much better is the optimal solution compared with, say, a random placement of the sources? The answer to this question is surprisingly simple and involves a small detour into some elementary number theory. Interestingly, the solution to this problem has connections to more advanced questions in numerical integration.

Our space of interest is the two-dimensional torus $\mathbb{T}^{2}$ represented as the square $[0,2 \pi]^{2}$ in which opposite boundary faces are glued together; see Figure 2 The aim is to find ways of effectively heating the torus $\mathbb{T}^{2}$. Suppose we are given $N$ identical radiators, how are we supposed to place them to guarantee that the temperature in $\mathbb{T}^{2}$ becomes everywhere nice and cozy as quickly as possible? We start by describing the heat given off by a radiator as a function $\phi: \mathbb{T}^{2} \rightarrow \mathbb{R}$. Such a function could, for example, have the form

$$
\phi(x, y)=\exp \left(-36\left(x^{2}+y^{2}\right)\right)
$$

which describes an initial temperature maximum at $(0,0)$ that decays exponentially with growing distance; see Figure 1.

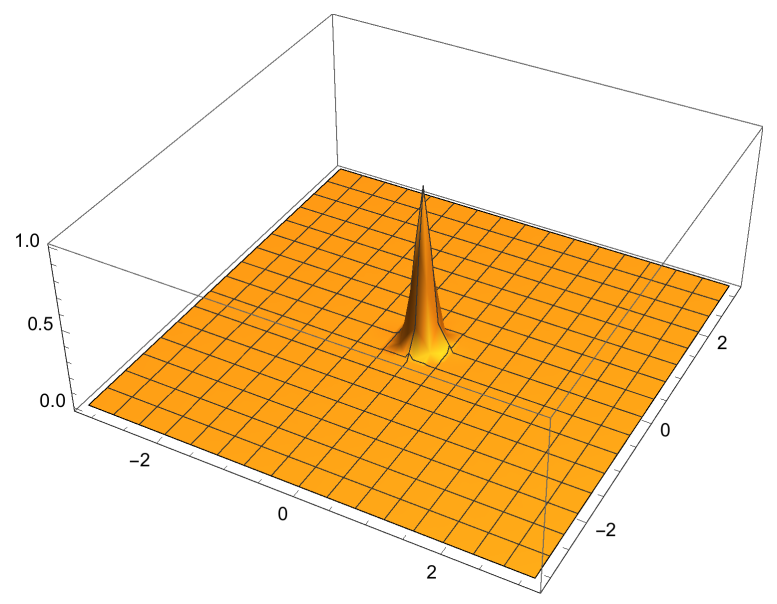

Figure 1. Plot of the function $\phi(x, y)=\exp \left(-36\left(x^{2}+y^{2}\right)\right)$.

We assume the $N$ radiators are placed at the points $\left(x_{n}, y_{n}\right)_{i=1}^{N}$, turned on for a little while and then turned off at time $t=0$. We then watch as the heat spreads throughout the room. Each radiator will have contributed a particular temperature distribution $\phi$ and, since all radiators are assumed to be identical, the temperature 
in $(x, y)$ at time $t=0$, denoted by $u(0, x, y)$, will be of the form

$$
u(0, x, y)=\sum_{n=1}^{N} \phi\left(x-x_{n}, y-y_{n}\right) .
$$

Denoting the temperature in $(x, y) \in \mathbb{T}^{2}$ at time $t$ by $u(t, x, y)$, the governing physical law is the free heat equation

$$
u_{t}=\Delta u
$$

(which we won't use directly). Physical intuition tells us that the heat is going to spread: points adjacent to warm points will heat up while, conversely, warm points surrounded by colder points will cool down. We expect the heat to be spread more and more evenly and that temperature will eventually converge to a constant (which can be explicitly computed because the total amount of heat in a closed system such as $\mathbb{T}^{2}$ has to stay constant):

$$
\forall(x, y) \in \mathbb{T}^{2} \quad \lim _{t \rightarrow \infty} u(t, x, y)=\frac{1}{\operatorname{area}\left(\mathbb{T}^{2}\right)} \int_{\mathbb{T}^{2}} u(0, x, y) d x d y .
$$

It turns out to be useful to rewrite $u(0, x, y)$ as a Fourier series

$$
u(0, x, y)=\sum_{(k, m) \in \mathbb{Z}^{2}} c_{k, m} e^{i(k x+m y)} .
$$

This representation immediately implies that

$$
\begin{aligned}
\frac{1}{\operatorname{area}\left(\mathbb{T}^{2}\right)} \int_{\mathbb{T}^{2}} u(0, x, y) d x d y & =\frac{1}{\operatorname{area}\left(\mathbb{T}^{2}\right)} \int_{\mathbb{T}^{2}}\left(\sum_{(k, m) \in \mathbb{Z}^{2}} c_{k, m} e^{i(k x+m y)}\right) d x d y \\
& =\frac{1}{\operatorname{area}\left(\mathbb{T}^{2}\right)}\left(\sum_{(k, m) \in \mathbb{Z}^{2}} c_{k, m} \int_{\mathbb{T}^{2}} e^{i(k x+m y)}\right) d x d y \\
& =c_{0,0},
\end{aligned}
$$

where $c_{0,0}$ is the constant term of the Fourier series of $u(0, x, y)$ because all the other integrals vanish. How should we pick the points $\left\{\left(x_{n}, y_{n}\right)_{n=1}^{N}\right\}$ to ensure that the initial condition $u(0, x, y)$ converges to the constant temperature as quickly as possible? We do not want to make any special assumptions on the form of $\phi: \mathbb{T}^{2} \rightarrow \mathbb{R}$. In particular, our placement rule will be valid for all smooth functions $\phi$ - even for heat distributions $\phi$ that are not radially symmetric or even physically meaningful. The key is to utilize elementary number theory in the form of particular permutations of finite fields.

\section{Result.}

Our argument will immediately show that every heat distribution converges to a constant with speed at least $\exp (-t)$. Moreover, we present a general and particularly nice placement of $N$ points (with $N$ prime) that uses number theory in an essential way to get a much faster convergence speed of at least $\exp (-(N / 4+\varepsilon) t)$ with $\varepsilon>0$ : for a prime number $N$, an integer $p$ satisfying $\sqrt{N} / 2<p \leq \sqrt{N}$ and an arbitrary $q \in \mathbb{N}$, we define the point set $\left\{\left(x_{n}, y_{n}\right)_{1 \leq n \leq N}\right\}$ with

$$
x_{n}=2 \pi \cdot \frac{n}{N} \quad \text { and } \quad y_{n}=2 \pi \cdot \frac{(p \cdot n+q) \bmod N}{N} ;
$$


Fig. 2 illustrates the construction for $N=7, p=2$ and $q=3$. Number theoretical constructions of point sets of this kind are called lattice rules and have a long history in the field of numerical integration (see, for example, the book by Sloan \& Joe [6]). It seems that the connection to the placement of radiators has not been observed before.

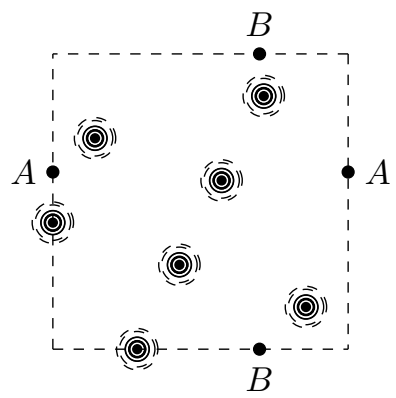

Figure 2. Placing seven (identical) heat distributions (radiators, candles, ...) at these points leads to convergence towards the constant room temperature with a speed of at least $e^{-4 t}$. The points $A$ and $B$ illustrate how boundary faces are identified. In other words, exiting to the left (up) makes you appear on the right (down).

Theorem 1. This set of points has the following property: for every smooth heat distribution $\phi: \mathbb{T}^{2} \rightarrow \mathbb{R}$, the initial distribution $u(0, x, y)$ given by

$$
u(0, x, y)=\sum_{n=1}^{N} \phi\left(x-x_{n}, y-y_{n}\right)
$$

converges towards the equilibrium with speed at least

$$
\max _{(x, y) \in \mathbb{T}^{2}}\left|u(t, x, y)-c_{0,0}\right| \leq c \cdot e^{-\alpha t},
$$

where $c_{0,0}$ is the constant term of the Fourier series of $u(0, x, y), c$ is a constant independent of $N$ and $t$, and $\alpha=\lfloor\sqrt{N} / 2\rfloor^{2}+2\lfloor\sqrt{N} / 2\rfloor+1 \geq N / 4$.

\section{HOW THIS WORKS.}

The argument comes in two parts: we use a neat formula for the heat equation to derive a condition on the points $\left\{\left(x_{n}, y_{n}\right)_{1 \leq n \leq N}\right\}$ that ensures that the heat equation converges quickly to its equilibrium state. In the second part of the argument, we verify that the condition is valid for our proposed set of points.

2.1. Step 1. It will be practical to use an explicit formula for the solution of the heat equation in terms of Fourier series. More precisely, if

$$
u(0, x, y)=\sum_{(k, m) \in \mathbb{Z}^{2}} c_{k, m} e^{i(k x+m y)},
$$

then (see e.g. [3]) the solution of the heat equation is given by

$$
u(t, x, y)=\sum_{(k, m) \in \mathbb{Z}^{2}} c_{k, m} e^{-\left(k^{2}+m^{2}\right) t} e^{i(k x+m y)},
$$


which can be easily verified by explicit computation since

$$
\frac{\partial}{\partial t} u(t, x, y)=\sum_{(k, m) \in \mathbb{Z}^{2}} c_{k, m}\left(-k^{2}-m^{2}\right) e^{-\left(k^{2}+m^{2}\right) t} e^{i(k x+m y)}=\Delta u(t, x, y) .
$$

This already shows that

$$
u(t, x, y)-\frac{1}{\operatorname{area}\left(\mathbb{T}^{2}\right)} \int_{\mathbb{T}^{2}} u(0, x, y) d x d y=\sum_{\substack{(k, m) \in \mathbb{Z}^{2} \\(k, m) \neq(0,0)}} c_{k, m} e^{-\left(k^{2}+m^{2}\right) t} e^{i(k x+m y)}
$$

can be written as the sum of exponential functions each of which decays at least as quickly as $e^{-t}$ independently of everything else. We now write the heat distribution of a single heat source (radiator, candle, ...) as

$$
\phi(x, y)=\sum_{(k, m) \in \mathbb{Z}^{2}} a_{k, m} e^{i(k x+m y)} .
$$

Plugging this into (1) and exchanging the order of summation leads to

$$
\begin{aligned}
\sum_{n=1}^{N} \phi\left(x-x_{n}, y-y_{n}\right) & =\sum_{n=1}^{N} \sum_{(k, m) \in \mathbb{Z}^{2}} a_{k, m} e^{i\left(k\left(x-x_{n}\right)+m\left(y-y_{n}\right)\right)} \\
& =\sum_{n=1}^{N} \sum_{(k, m) \in \mathbb{Z}^{2}} a_{k, m} e^{-i k x_{n}} e^{-i m y_{n}} e^{i(k x+m y)} \\
& =\sum_{(k, m) \in \mathbb{Z}^{2}} \underbrace{a_{k, m}\left(\sum_{n=1}^{N} e^{-i k x_{n}} e^{-i m y_{n}}\right)}_{:=c_{k, m}} e^{i(k x+m y)} .
\end{aligned}
$$

The formula for the heat equation tells us that we would like to pick the points $\left\{\left(x_{n}, y_{n}\right)_{n=1}^{N}\right\}$ such that the expression in brackets vanishes for as many small values of $k, m$ as possible. More precisely, we obtain the following Lemma.

Lemma 1. If the set of points $\left(x_{n}, y_{n}\right)_{1 \leq n \leq N}$ has the property that

$$
\sum_{n=1}^{N} e^{i k x_{n}} e^{i m y_{n}}=0
$$

for all $(k, m) \in \mathbb{Z}^{2}$ with $(k, m) \neq(0,0),|k|,|m| \leq \ell$ and $\ell \in \mathbb{Z}$, then for all smooth $\phi: \mathbb{T}^{2} \rightarrow \mathbb{R}$ and corresponding $u(0, x, y)$ as in (1) we have, for some constant $c>0$,

$$
\max _{(x, y) \in \mathbb{T}^{2}}\left|u(t, x, y)-\frac{1}{\operatorname{area}\left(\mathbb{T}^{2}\right)} \int_{\mathbb{T}^{2}} u(0, x, y) d x d y\right| \leq c \cdot e^{-\left(\ell^{2}+2 \ell+1\right) t} .
$$

Proof. We have the explicit solution to the heat equation as

$$
u(t, x, y)=\sum_{(k, m) \in \mathbb{Z}^{2}} c_{k, m} e^{-\left(k^{2}+m^{2}\right) t} e^{i(k x+m y)},
$$

where $c_{k, m}=0$ for all $|k|,|m| \leq \ell, \ell \in \mathbb{Z}$. This means that the smallest sum of squares $k^{2}+m^{2}$ of a pair $(k, m)$ for which $c_{k, m} \neq 0$ is at least $(\ell+1)^{2}+0^{2}=\ell^{2}+2 \ell+1$ for the pair $(\ell+1,0)$. Hence, the first nonzero exponential term decays at least as 
quickly as $e^{-\left(\ell^{2}+2 \ell+1\right) t}$. As for nonzero coefficients, we can use the fact that $\phi$ is smooth to conclude that

$$
\left|a_{k, m}\right|=\left|\frac{1}{\operatorname{area}\left(\mathbb{T}^{2}\right)} \int_{\mathbb{T}^{2}} \phi(x, y) e^{-i(k x+m y)} d x d y\right| \leq \max _{(x, y) \in \mathbb{T}^{2}}|\phi(x, y)|
$$

and classical results (see e.g. [3]) on the decay of Fourier coefficients of smooth functions to ensure that everything is summable.

2.2. Step 2. We will now show that our point set has the property from the lemma.

Lemma 2. For a prime $N$, let $\left\{\left(x_{n}, y_{n}\right): 1 \leq n \leq N\right\}$ be as defined above. Then for $(0,0) \neq(k, m) \in \mathbb{Z}^{2}$ with $|k|,|m| \leq \sqrt{N} / 2$

$$
\sum_{n=1}^{N} e^{i k x_{n}} e^{i m y_{n}}=\sum_{n=1}^{N} e^{2 \pi i \frac{k \cdot n+m(p \cdot n+q)}{N}}=\sum_{n=1}^{N} e^{2 \pi i \frac{(k+m \cdot p) \cdot n+m \cdot q}{N}}=0 .
$$

Proof. This is a fairly intricate expression. We want to relate it to the fact that the $N$-th roots of unities sum to 0 because they form a geometric progression, i.e.

$$
\sum_{n=1}^{N} e^{2 \pi i \frac{n}{N}}=\sum_{n=1}^{N}\left(e^{2 \pi i \frac{1}{N}}\right)^{n}=\frac{e^{2 \pi i \frac{N+1}{N}}-1}{e^{2 \pi i \frac{1}{N}}-1}-1=0 .
$$

Therefore, if $n \rightarrow(k+m \cdot p) \cdot n+m \cdot q$ is a bijection on $\mathbb{Z}_{N}=\{0,1, \ldots, N-1\}$, then (4) would imply (3) since we are still summing over the roots of unity (just in a different order); see Figure 3. For which values of $k$ and $m$ is this the case?

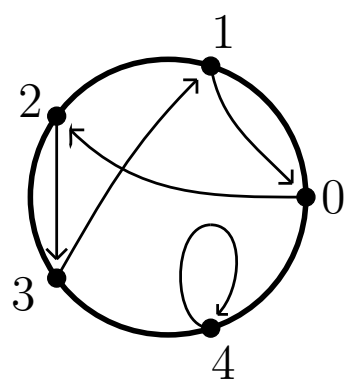

Figure 3. The map $n \rightarrow 3 n+2$ is a bijection on $\mathbb{Z}_{5}$ and induces a reordering of the $5-$ th roots of unity.

First, it is clear that the map $n \rightarrow(k+m \cdot p) \cdot n+m \cdot q$ is a bijection on $\mathbb{Z}_{N}$ if and only if $n \rightarrow(k+m \cdot p) \cdot n$ is a bijection on $\mathbb{Z}_{N}$. Now, since $N$ is prime, the map $n \rightarrow(k+m \cdot p) \cdot n$ is a bijection if and only if

$$
N \nmid m \cdot p+k,
$$

which is true for $k, m$ and $p$, as assumed. Indeed, since

$$
|k|,|m| \leq \frac{1}{2} \sqrt{N}<p \leq \sqrt{N}
$$

we have

$$
|m \cdot p+k| \leq|m \cdot p|+|k| \leq \frac{1}{2} N+\frac{1}{2} \sqrt{N}<N .
$$


This means that $N \mid m \cdot p+k$ only if $m \cdot p+k=0$. However, $|k|<p$ imlies $-p<k<p$ and so

$$
(m-1) p<m \cdot p+k<(m+1) p .
$$

Thus $m \cdot p+k=0$ implies $m-1<0<m+1$, which gives $m=0$ and thus $k=0$. But this contradicts the assumption that $(k, m) \neq(0,0)$, thus we must have $N \nmid m \cdot p+k$, as claimed.

Since $k, m \in \mathbb{Z}$ and $|k|,|m| \leq \sqrt{N} / 2$, we set $\ell=\lfloor\sqrt{N} / 2\rfloor$ in Lemma 1 to obtain the result stated in Theorem 1 .

\section{EXAMPLES.}

To illustrate our result we place 7 radiators as shown in Figure 2 and use the heat distribution $\phi$ from the introduction. It turns out that $\phi$ has a particularly nice Fourier series, i.e.,

$$
\phi(x, y)=\exp \left(-36\left(x^{2}+y^{2}\right)\right)=\sum_{(k, m) \in \mathbb{Z}^{2}} a_{k, m} e^{i(k x+m y)},
$$

with

$$
a_{k, m}=\frac{1}{4 \cdot 36 \cdot \pi} \cdot \exp \left(-\frac{k^{2}+m^{2}}{4 \cdot 36}\right) .
$$

The coefficients $a_{k, m}$ are all we need to build the functions $u(0, x, y)$ and $u(t, x, y)$. We observe that the average value of one heat source is $a_{0,0}$ and thus, by summation, $c_{0,0}=N a_{0,0}$. This shows that the average temperature is

$$
\frac{1}{\operatorname{area}\left(\mathbb{T}^{2}\right)} \int_{\mathbb{T}^{2}} u(0, x, y) d x d y=7 a_{0,0}=\frac{7}{144 \pi} \approx 0.01547 \ldots
$$

Thus, we can numerically investigate how fast $\max _{(x, y)} u(t, x, y)$ converges to $7 a_{0,0}$ as $t \rightarrow \infty$ for different point sets. According to Lemma 2 all coefficients $c_{k, m}$ with $|k|,|m| \leq \sqrt{7} / 2$ vanish. Hence, setting $\ell=\lfloor\sqrt{7} / 2\rfloor$ in Lemma 1 , we obtain a convergence of at least $e^{-4 t}$, which can be also observed from our numerical results in Figure 4 This can be easily compared to random points: for a set of $N$ random points, we expect a speed of convergence of $(c / \sqrt{N}) e^{-t}$, where $c$ is a constant depending only on $\phi$. This shows that there is quite a bit of decay for $t$ small but, as $t$ becomes large, the result is much worse than our construction.

\section{The Big Picture.}

To illustrate the generality of the ideas in $\S 3$, we outline the appearance of these number theoretical constructions in two more situations.

4.1. Fourier Analysis. A result of Montgomery [4, 5] (proven for a quite different purpose) implies that our construction is optimal up to a constant: $N$ heat sources can never decay faster than $e^{-\alpha t}$ where $\alpha \sim N$. A result [7] of the second author implies that this is even true if we allow the heat sources to have different (positive weights). Statements of this type are probably true on very general domains, the best known result in that direction is [1]. 


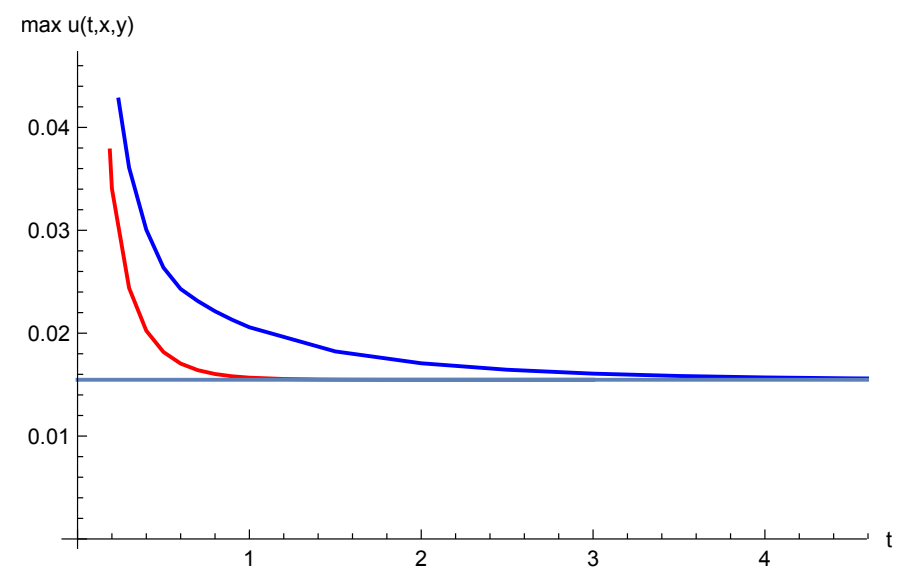

Figure 4. Comparison of convergence of $\max _{(x, y)} u(t, x, y)$ for different point sets. The red graph shows the convergence for our point set (with convergence speed $e^{-4 t}$ ), the blue graph corresponds to a set of 7 random points, which almost surely will always only converge with speed $e^{-t}$.

4.2. Cubature formulas. A set of $N$ points $\left\{\left(x_{n}, y_{n}\right)_{n=1}^{N}\right\}$ is said to be an exact cubature formula of degree $\ell$ on $\mathbb{T}^{2}$ if we can compute the integral of every function of the form

$$
f(x, y)=\sum_{\substack{(k, m) \in \mathbb{Z}^{2} \\|k|+|m| \leq \ell}} a_{k, m} e^{i(k x+m y)}
$$

exactly by taking the average value at the $N$ points, i.e. if for all such $f$

$$
\frac{1}{(2 \pi)^{2}} \int_{\mathbb{T}^{2}} f(x, y) d x d y=a_{0,0}=\frac{1}{N} \sum_{n=1}^{N} f\left(x_{n}, y_{n}\right) .
$$

Plugging in and exchanging the order of summation gives

$$
\sum_{n=1}^{N} f\left(x_{n}, y_{n}\right)=\sum_{\substack{(k, m) \in \mathbb{Z}^{2} \\|k|+|m| \leq \ell}} a_{k, m}\left(\sum_{n=1}^{N} e^{i k x_{n}} e^{i m y_{n}}\right)
$$

and, hence the similarity. The underlying expression is the same (and the goal is to select points that make it vanish for all $|k|+|m| \leq \ell$ with $(k, m) \neq(0,0))$. Interestingly, Cools \& Sloan [2] have discovered cubature formulas with the minimal possible number of points for a given $\ell$ that are not lattice rules. It would be interesting to see similar results in our context.

4.3. Wave equation. The radiator problem also appears in other physical problems: suppose we have a $\mathbb{T}^{2}$-swimming pool and want to create waves by throwing in $N$ stones simultaneously. The goal is to pick the points in such a way that we get no low-frequency waves at all. For simplicity, we can model this with the wave equation

$$
u_{t t}=\Delta u \quad \text { and }\left.\quad u_{t}\right|_{t=0}=0
$$


for $u(0, x, y)$ as in (1). Using again the theory of Fourier series, we follow our line of thought from Step 1 in $\S 3$. We solve the wave equation explicitly with the formula

$$
u(t, x, y)=\sum_{(k, m) \in \mathbb{Z}^{2}} c_{k, m} e^{i\left(k^{2}+m^{2}\right) t} e^{i(k x+m y)} .
$$

and see by the same subsequent computation, that, in order not to generate lowfrequency waves, it is required that 2 holds for as many small $(k, m) \in \mathbb{Z}^{2}$ as possible. These point sets and the same arguments even apply to more complicated equations such as the Schrödinger equation $i u_{t}=\Delta u$.

\section{REFERENCES}

[1] D. Bilyk, F. Dai and S. Steinerberger, General and Refined Montgomery Lemmata, arXiv:1801.07701

[2] R. Cools and I. Sloan, Minimal cubature formulae of trigonometric degree. Math. Comp. 65 (1996), no. 216, 1583-1600.

[3] L. Evans, Partial differential equations. Graduate Studies in Mathematics, 19. American Mathematical Society, Providence, RI, 1998. xviii+662 pp. ISBN: 0-8218-0772-2

[4] Irregularities of distribution by means of power sums. Proceedings of the Congress on Number Theory (Spanish) (Zarauz, 1984), 11-27

[5] H. Montgomery, Ten Lectures at the Interface of Harmonic Analysis and Number Theory, American Mathematical Society, 1994.

[6] I. Sloan and S. Joe, Lattice methods for multiple integration. Oxford Science Publications. The Clarendon Press, Oxford University Press, New York, 1994. xii+239 pp. ISBN: 0-19-853472-8

[7] S. Steinerberger, Spectral Limitations of Quadrature Rules and Generalized Spherical Designs, arXiv:1708.08736

Summary. We discuss an amusing application of number theory: suppose you find yourself on the two-dimensional torus $\mathbb{T}^{2}$ equipped with $N$ candles and want to position the candles in such a way that they heat up the room as efficiently as possible. To achieve this goal, we give a construction that uses number theory as the main ingredient and explain related results.

Florian Pausinger(MR920909) is a lecturer at Queen's University Belfast. He studied pure mathematics at the University of Salzburg, Austria, before he moved to Vienna where he received his PhD from IST Austria under the direction of Herbert Edelsbrunner. In his research he is mainly interested in the very basic question of how to distribute points in a uniform manner in squares, on spheres or on more complicated shapes. Moreover, he is interested in waves - both, in classroom teaching fluid mechanics as well as on the Irish beaches while surfing. School of Mathematics and Phyics, Queen's University, University Rd, Belfast BT7 1NN, UK. f.pausinger@qub.ac.uk

Stefan Steinerberger (MR869041) received his undergraduate education in Austria and a PhD in Mathematical Analysis from Bonn, Germany. He then moved to Yale where he is an Assistant Professor of Mathematics. He spends most of his days trying to figure out why things vibrate the way they do (not so easy!!) and drinking coffee. Department of Mathematics, Yale University, 10 Hillhouse Avenue, New Haven, CT 06511, USA. stefan.steinerberger@yale.edu 